\title{
Biomechanics of the Lumbar Spine After Dynamic Stabilization
}

\author{
Chiara Maria Bellini, MSEng, ${ }^{*} \dagger$ Fabio Galbusera, MSEng, $+\$$ Manuela T. Raimondi, PhD, $*$ \\ Giuseppe V. Mineo, MD, $\&$ and Marco Brayda-Bruno, $M D^{*}$
}

\begin{abstract}
Target of the study was to predict the biomechanics of the instrumented and adjacent levels due to the insertion of the DIAM spinal stabilization system (Medtronic Ltd). For this purpose, a 3-dimensional finite element model of the intact L3/ S1 segment was developed and subjected to different loading conditions (flexion, extension, lateral bending, axial rotation). The model was then instrumented at the L4/L5 level and the same loading conditions were reapplied. Within the assumptions of our model, the simulation results suggested that the implant caused a reduction in range of motion of the instrumented level by $17 \%$ in flexion and by $43 \%$ in extension, whereas at the adjacent levels, no significant changes were predicted. Numerical results in terms of intradiscal pressure, relative to the intact condition, predicted that the intervertebral disc at the instrumented level was unloaded by $27 \%$ in flexion, by $51 \%$ in extension, and by $6 \%$ in axial rotation, while no variations in pressure were caused by the device in lateral bending. At the adjacent levels, a change of relative intradiscal pressure was predicted in extension, both at the L3/L4 level, which resulted unloaded by $26 \%$ and at the L5/S1 level, unloaded by $8 \%$. Furthermore, a reduction in terms of principal compressive stress in the annulus fibrosus of the L4/L5 instrumented level was predicted, as compared with the intact condition. These numerical predictions have to be regarded as a theoretical representation of the behavior of the spine, because any finite element model represents only a simplification of the real structure.
\end{abstract}

Key Words: dynamic stabilization, soft stabilization, finite element, lumbar biomechanics

(J Spinal Disord Tech 2007;20:423-429)

T raditionally, spinal fusion has been the gold standard of surgical approaches to the management of low back pain. For 2 decades, the dominant surgical

Received for publication July 28, 2006; accepted December 13, 2006.

From the *IRCCS Istituto Ortopedico Galeazzi; LaBS, Departments of $\dagger$ Bioengineering; $\ddagger$ Structural Engineering, Politecnico di Milano; and $\S$ Department of Clinical Sciences at L. Sacco Hospital, Università degli Studi di Milano, Milan, Italy.

Funded in part by Medtronic Ltd, through a research fellowship.

Reprints: Chiara Maria Bellini, MSEng, Bioengineering Unit, IRCCS Istituto Ortopedico Galeazzi, via R.Galeazzi 4, 20161 Milan, Italy (e-mail: chiara.bellini@polimi.it).

Copyright (C) 2007 by Lippincott Williams \& Wilkins justification for fusing a segment has been the concept of instability. ${ }^{1}$ The term instability would imply an abnormal motion under physiologic $\operatorname{load}^{2}$ but, despite considerable efforts over many years, no clear relationship has been established between low back pain and abnormal motion. ${ }^{2}$ Although abnormal motion is seen radiologically in many cases of disc degeneration, it is not always present in symptomatic disc degeneration. Even when it is present, pain may not be consistent. It is therefore difficult to find any basis for the concept of abnormal movement or instability as a cause of back pain. $^{2}$

Low back symptoms often implicate abnormal loading rather than motion as a primary source of pain. ${ }^{1}$ Many patients complain of postural or positional pain as a prevailing symptom. ${ }^{3}$ Under physiologic conditions, the disc spreads load evenly over the vertebral endplates. After disc degeneration, load distribution across the endplates becomes uneven, with areas of high spot loading, particularly associated with certain positions. The high point loadings of the disc will lead to similar focal loading of the adjacent vertebral endplates, containing sensory nerve endings, and possibly leading to pain. This has been confirmed by in vivo stress profilometry studies reported by McNally et $\mathrm{al}^{4}{ }^{4}$ They demonstrated that the pattern of loading, rather than the absolute levels of loading, was related to pain generation in the degenerated spine. This may help to explain the lack of correlation between degrees of disc degeneration and back pain, because individual anatomic and consequential load transmission changes vary highly from one person to another. ${ }^{1}$

These observations suggest that low back pain may have etiologies related to load, and successful treatment may exist beyond fusion. Altering the load transmission across the degenerated disc may therefore be beneficial. Furthermore, such benefit could be accomplished without the elimination of movement. Dynamic stabilization devices may change load transmission to get more tolerable positions, and may limit motion to reduce painful positions. $^{2}$ In other words, such devices are able to control the segment motion and to share the load with the disc, becoming a load-bearing system.

The various dynamic stabilization devices that have been described in the spinal literature and used clinically are all posterior implants. The DIAM spinal stabilization system (Medtronic Ltd, Memphis, TN) is a silicone 
TABLE 1. Anisotropic Properties of Vertebral Cancellous Bone Assumed for the FE Model, in a Cartesian Coordinate System $x, y, z$

\begin{tabular}{lrll}
\hline \multicolumn{1}{c}{ Modulus (MPa) } & \multicolumn{2}{c}{ Poisson Ratio } \\
\hline$E_{z}$ & 340 & $v_{\mathrm{xz}}=v_{\mathrm{yz}}$ & 0.1 \\
$E_{x}=E_{y}$ & 112 & $v_{\mathrm{xy}}$ & 0.3 \\
$G_{y z}=G_{z x}$ & 53 & & \\
$G_{x y}$ & 52 & & \\
\hline
\end{tabular}

interspinous spacer, covered by a polyethylene terephthalate coat, secured in place by 2 ligatures connecting the device to the spinous processes of the instrumented segment.

Target of the present study was to predict the biomechanics of the instrumented and adjacent levels after the insertion of this interspinous implant. For this purpose, a 3-dimensional finite element (FE) model of the intact $\mathrm{L} 3 / \mathrm{S} 1$ segment was developed and subjected to different loading conditions (flexion, extension, lateral bending, axial rotation). The model was then instrumented at the L4/L5 level and the same loading condition was reimposed. Simulation results are presented in terms of range of motion (ROM), intradiscal pressure, and principal compressive stress. The advantages, disadvantages, and limitations of the method are presented and discussed.

\section{MATERIALS AND METHODS}

A 3-dimensional FE model of the intact $\mathrm{L} 3 / \mathrm{S} 1$ segment was developed. The 3-dimensional geometry was reconstructed from computed tomography (CT) scans taken from the Visible Human Dataset, a collection of radiographic images and photos of sections of the whole human body made available by the American National Institute of Health. A commercial software (Amira, TGS, San Diego, CA) was used to transform planar CT scans into a solid model of the lumbar segment. The 3dimensional geometry was exported to a FE preprocessor software (Gambit, Fluent Inc, Lebanon, NH). The intervertebral discs were represented as continuum structures occupying the intervertebral spaces. Each structure was subdivided in an inner volume representing the nucleus pulposus and an external layer representing the annulus fibrosus. The geometrical entities were then
TABLE 3. Anisotropic Properties of Annulus Fibrosus Assumed for the FE Model in a Cylindrical Coordinate System $r, t, z$

\begin{tabular}{lrll}
\hline \multicolumn{2}{c}{ Modulus (MPa) } & \multicolumn{3}{c}{ Poisson Ratio } \\
\hline$E_{r}$ & 35 & $v_{\mathrm{rz}}$ & 0.35 \\
$E_{t}$ & 8 & $v_{\mathrm{rt}}$ & 1.2 \\
$E_{z}$ & 2 & $v_{\mathrm{tz}}$ & 0.25 \\
$G_{r t}=G_{t z}=G_{r z}$ & 1 & & \\
\hline
\end{tabular}

discretized into linear tetrahedral elements. The thin cortical shell, which surrounds the vertebral bodies was modeled using triangular shell elements of $0.4 \mathrm{~mm}$ in thickness.

Considering its highly oriented structure, optimized to resist axial loads, the trabecular vertebral bone was modeled as linear elastic orthotropic transversely isotropic (Table 1). ${ }^{5-7}$ The posterior elements of the vertebrae were modeled as linear elastic isotropic, with elastic modulus $3.5 \mathrm{GPa}$ and Poisson ratio 0.25 (Table 2). ${ }^{8}$ Cortical bone was modeled as isotropic linear elastic, with elastic modulus $12 \mathrm{GPa}$ and Poisson ratio $0.3{ }^{8}$ The annulus was modeled as a linear elastic cylindrical orthotropic material (Table 3), with longitudinal axis passing through the upper and lower endplate centers. The nucleus pulposus was modeled as an almostincompressible continuum, with elastic modulus $1 \mathrm{MPa}$ and Poisson ratio $0.499 .^{9}$ The ligaments were modeled as nonlinear springs ${ }^{10}$ and were assumed to sustain tensile force only. The ligaments included in the model were: anterior longitudinal, posterior longitudinal, flavum, intertransverse, interspinous, supraspinous, and capsular. To include the mechanical contribution of the facet joints, a contact condition was defined between the surfaces of articular facets, with friction coefficient of 0.01. Static simulations were carried out in flexion, extension, lateral bending, and axial rotation, using the commercial FE code ABAQUS 6.4 (ABAQUS Inc, Providence, RI). To impose the moments, a set of concentrated forces acting on several nodes on the upper endplate of L3 was defined. The value of each force was determined to make the force system result in a moment of $10 \mathrm{Nm}$ value and satisfy the translational equilibrium, in the prescribed directions. The inferior surface of the S1 vertebra was fixed. Simulation results were properly validated against literature data.

TABLE 2. Element Type and Material Properties Assumed for the FE Model

\begin{tabular}{llcc}
\hline Part & Element Type & Modulus (MPa) & Poisson Ratio \\
\hline Cancellous bone & Linear tetrahedron & Table 1 & Table 1 \\
Cortical bone & Linear shell & 12000 & 0.3 \\
Posterior elements & Linear tetrahedron & 3500 & 1 \\
Nucleus pulposus & Linear tetrahedron & Table 3 & 0.25 \\
Annulus fibrosus & Linear tetrahedron & Nonlinear & 0.499 \\
Ligaments & Non-linear spring & 20 & Table 3 \\
DIAM core & Linear tetrahedron & 5000 & 0.45 \\
DIAM ligatures & Truss & & 0.3 \\
\hline
\end{tabular}




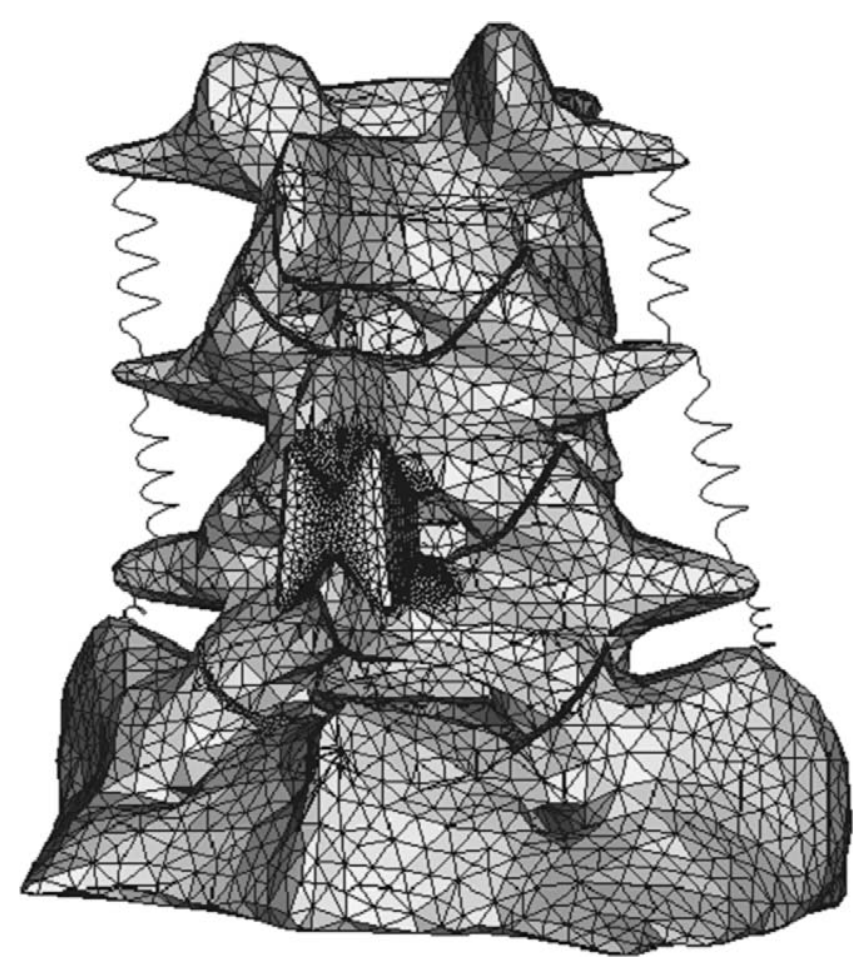

FIGURE 1. Three-dimensional FE model of the instrumented L3/S1 segment.

The FE model of the intact lumbar segment was then modified to simulate the biomechanics of the L3/S1 segment after implantation of the DIAM spinal stabilization system (Fig. 1). An adequate device size (height $8 \mathrm{~mm}$ ) was chosen by analyzing the CT scans of the spinal segment. A 3-dimensional model of the device was built and included into the FE model of the intact segment at the L4/L5 level. According to the surgical procedure, the interspinous ligament at the instrumented level was removed. To obtain congruent contact surfaces, a Boolean operation was performed between the posterior elements at the L4/L5 level and the device. The implant was discretized into linear tetrahedral elements, while the ligatures were modeled as truss elements connecting the device to the posterior elements of the L4/L5 segment. A friction contact was assumed at the bone/implant interface. Static simulations were carried out by assuming the same loading and boundary conditions assumed for the intact model.

Rotation values (ROMs of the functional units) were extracted from the simulations for both the intact and the instrumented model, for all loading conditions and at all spinal levels. To evaluate the ability of the device to unload the intervertebral disc, the ratio between the average intradiscal pressure of the instrumented configuration, $P$, and those of the intact configuration, $P_{0}$, was calculated for all load configurations. Furthermore, the values of principal compressive stress of the L4/ L5 annulus fibrosus were calculated, for the intact and the instrumented model in extension.

\section{RESULTS}

In the motion from the neutral to the flexed position, our numerical results predict that the mechanical interaction of the implant with the spine segments reduces the ROM of the instrumented level by $17 \%$, whereas the ROMs at the adjacent levels are not affected (Fig. 2). In the neutral to extended position, the ROM at the L4/L5 level is reduced by $43 \%$, whereas at the adjacent levels the ROM is reduced by $11 \%$ and by $7 \%$, at the L3/L4 and L5/S1 respectively (Fig. 3). No difference in the calculated ROMs is found during lateral bending and axial rotation, as compared with the intact condition, both at the instrumented and the adjacent levels (Figs. 4, 5).

The calculated values of relative average intradiscal pressure are shown in Figure 6. Our numerical results predict that the intervertebral disc at the instrumented level is unloaded by $27 \%$ in flexion, by $51 \%$ in extension, and by $6 \%$ in axial rotation. At the adjacent levels, in extension, a change of relative intradiscal pressure is predicted, both at the L3/L4 level, which resulted unloaded by $26 \%$ and at the L5/S1 level, unloaded by $8 \%$.

Numerical results in terms of principal compressive stress predict a reduction in the posterior part of the annulus fibrosus of the L4/L5 segment, for the instrumented model, as compared with the intact one (Fig. 7).

\section{DISCUSSION}

Target of the present study was to investigate the biomechanics of the instrumented and adjacent levels

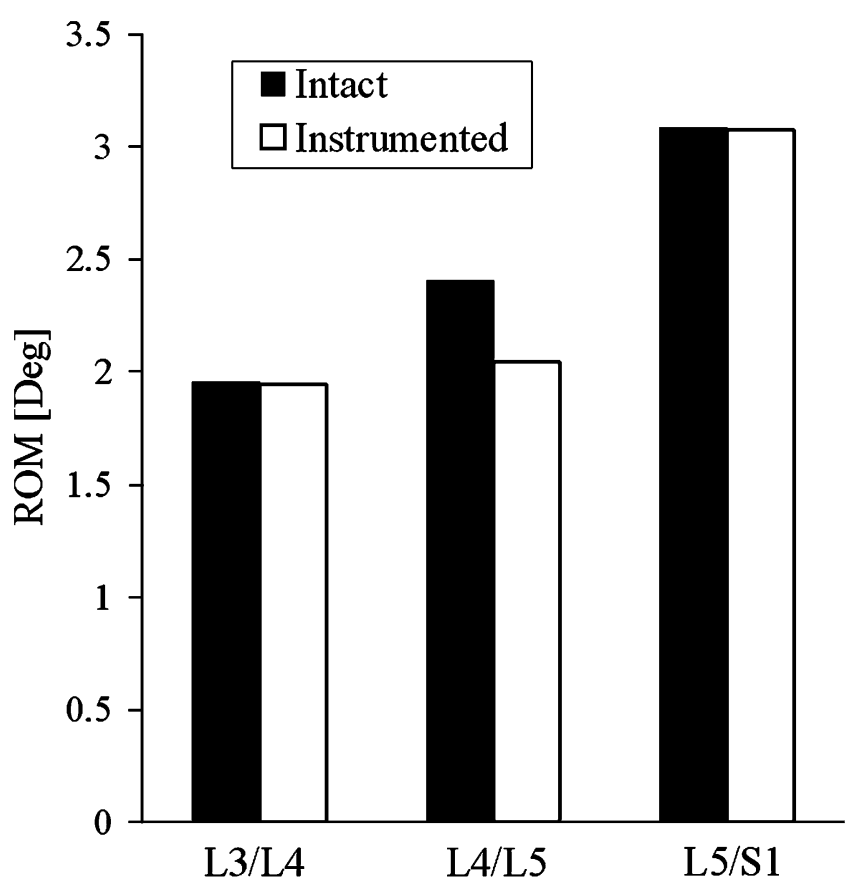

FIGURE 2. Intact and instrumented segmental ROM under $10 \mathrm{Nm}$ flexion moment. The L4/L5 segment is the instrumented level. 


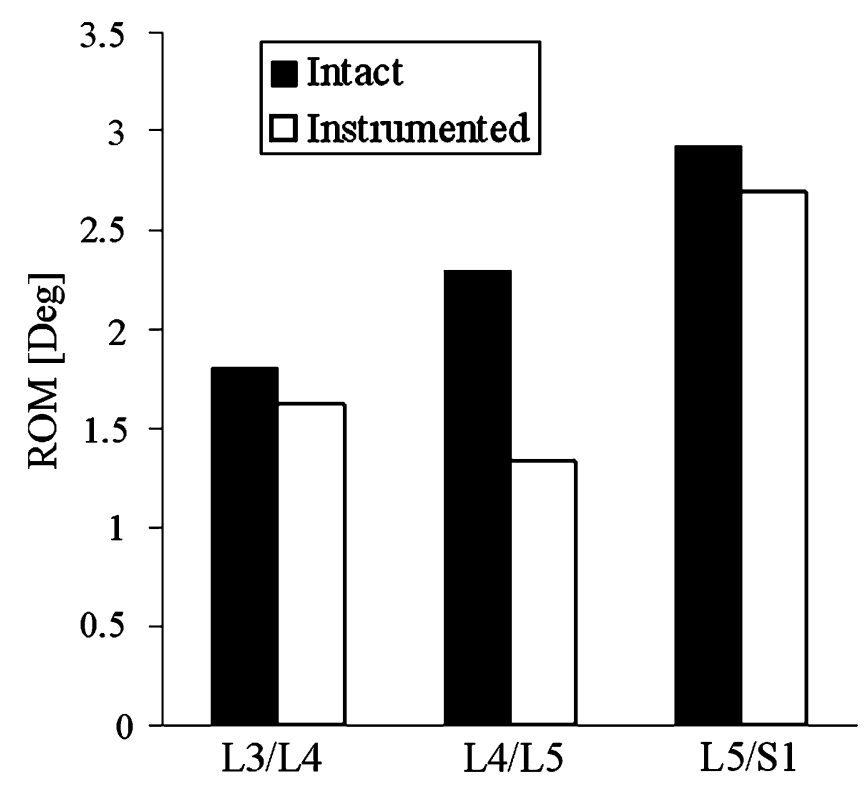

FIGURE 3. Intact and instrumented segmental ROM under $10 \mathrm{Nm}$ extension moment. The L4/L5 segment is the instrumented level.

owing to the insertion of the DIAM spinal stabilization system. For this purpose, a 3-dimensional FE model of the intact L3/S1 segment was developed and properly validated against literature data, considering different loading conditions: flexion, extension, lateral bending,

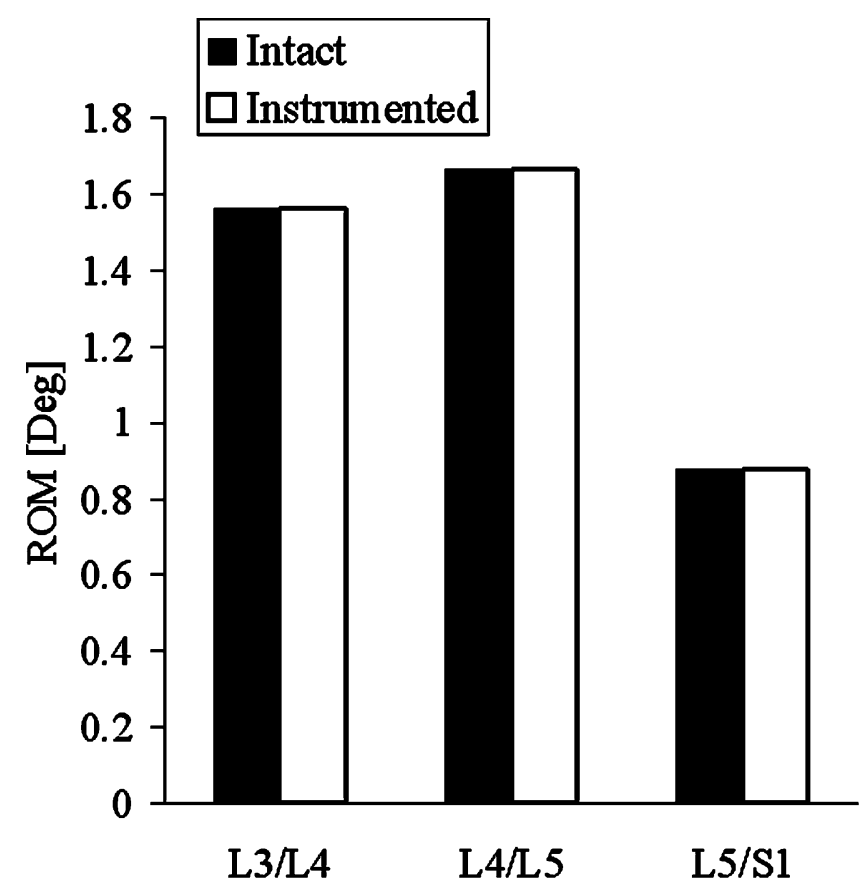

FIGURE 4. Intact and instrumented segmental ROM under $10 \mathrm{Nm}$ lateral bending moment. The L4/L5 segment is the instrumented level.

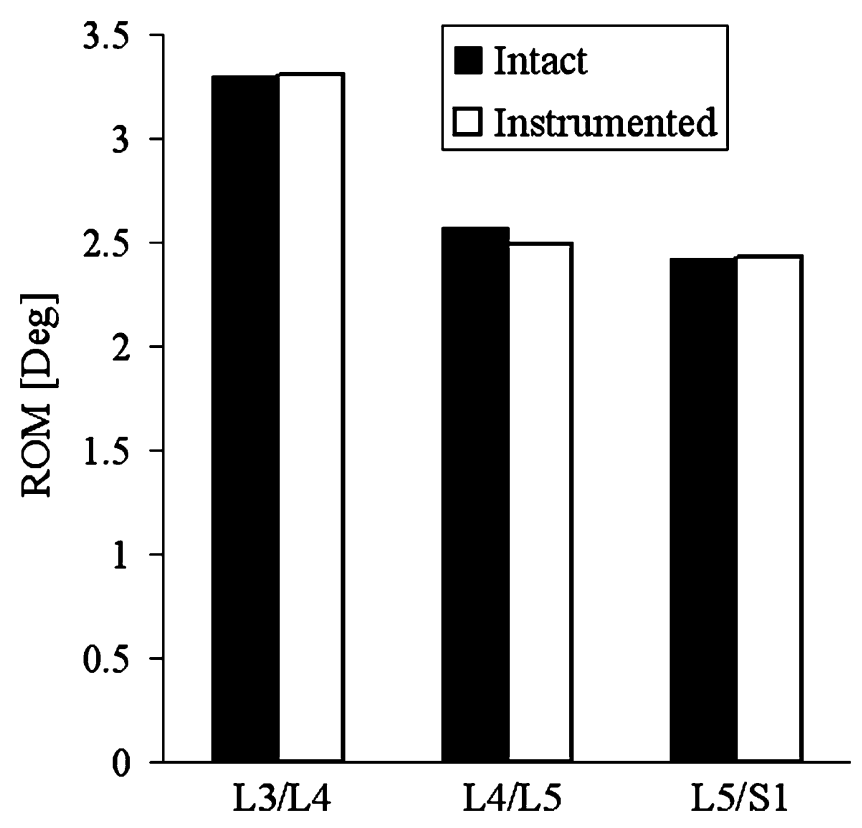

FIGURE 5. Intact and instrumented segmental ROM under $10 \mathrm{Nm}$ axial torque moment. The L4/L5 segment is the instrumented level.

and axial rotation. The model was instrumented, by including the device at the L4/L5 level and the same loading conditions were reapplied. Our numerical results predict that, at the instrumented level, the mechanical effect of the implant is to reduce the ROM and to unload the intervertebral disc in flexion and in extension.

Many computational studies on lumbar spine mechanics have been carried out by using the FE method. Compared with experimental investigations, the FE method has the advantage of enabling easy modification of individual parameters without the need for new specimens. When numerical results are compared with experimental data with subsequent adjustment of the model, the FE method becomes a powerful tool for analyzing biomechanical problems. Nevertheless, because FE models represent only a simplification of the real structure, because of many assumptions, which have to be made, caution must be adopted when interpreting the numerical results. The numerical results reported in our study have to be taken as predictions within the assumptions of our model.

The numerical results obtained in the current study for the intact model are consistent within the range of experimental data from literature. ${ }^{11-13}$ No comparison can be made for the instrumented model simulation results, owing to the lack of previous experimental and computational studies.

The numerical results of the present study predict that the kinematics of the instrumented level during the flexion and the extension movement is affected by the mechanical interaction with the device. One reason that significant changes were not observed at the adjacent levels during flexion-extension may be that the interspinous implant is not a rigid device, such as those used in 


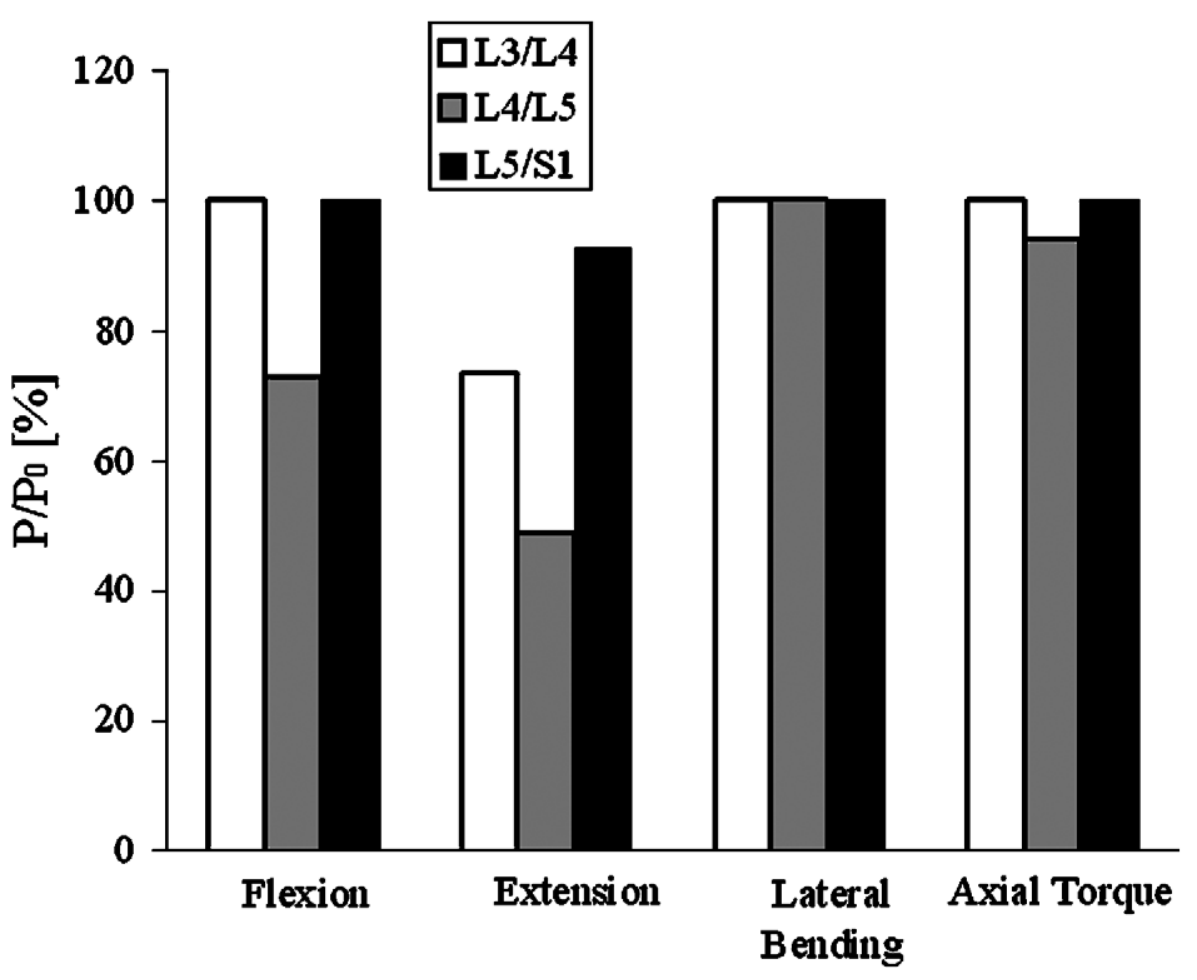

fusion, and still allows about 3 degrees of rotation, mostly in flexion.

Our numerical results predict that the mechanical interaction of the device with the spine segments unloads the disc, both in flexion and in extension, at the instrumented level (Figs. 6, 7). This means that part of the load is shifted posteriorly and shared with the disc by the device, which becomes a load-bearing system. In a degenerated condition, the disc is depressurized and, consequently, the annulus results over-loaded probably leading to a painful condition. The implant may be able to share part of the load, unloading the annulus and helping to relieve pain.

During extension, our numerical results predict a reduction in terms of intradiscal pressure in the upper intact segment L3/L4 (Fig. 6). This undesired decrease might determine an increased load transmission through the facet joints, possibly leading to a degeneration process. In such a condition, larger loads may be transmitted also through the annulus. Overloading the annulus, unprotected by the supporting pressure of the nucleus, could lead to splitting and inward folding of the annulus itself. Areas of main load transmission across the disc become dependent on the posture (in flexion the anterior annulus, in extension the posterior annuls) eventually leading to a painful condition.

In the spinal fusion literature, restriction of segment motion has been shown to result in abnormal motion of the adjacent levels, possibly leading to degeneration. ${ }^{14-16}$ Previous studies have reported changes in the kinematics of the adjacent segments after fusion. ${ }^{17-19}$ An increase in the motion of the upper intact segment during combined compression and bending loads was reported by Lee and Langrana. ${ }^{17}$ However, Yang et $\mathrm{al}^{20}$ did not find a
FIGURE 7. Principal compressive stress of the L4/L5 annulus fibrosus during extension, for the intact (A) and the instrumented model (B).
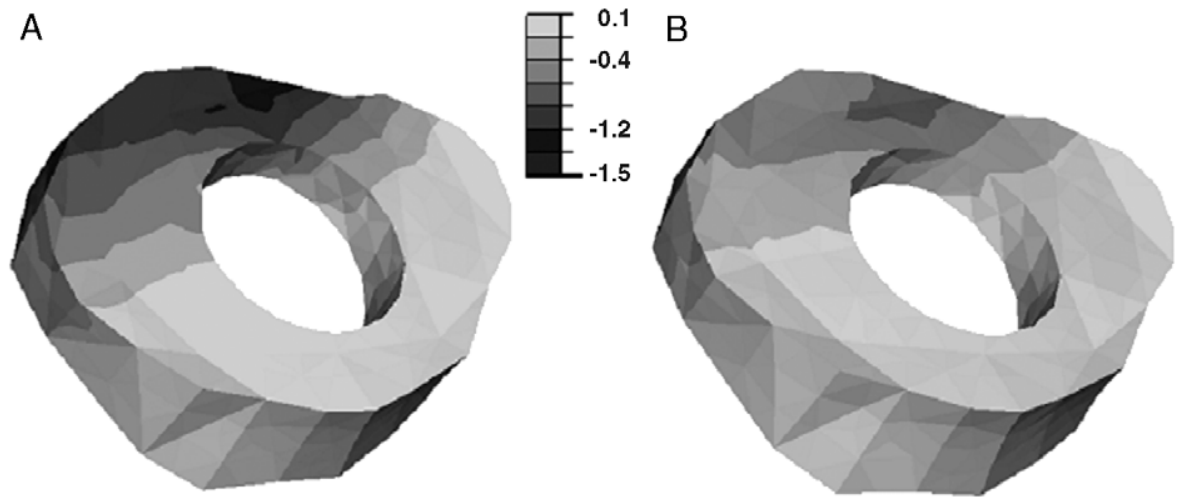
remarkable increase in the stress on the adjacent level during combined compression-torsion loading. Chow et $\mathrm{al}^{18}$ reported an increase in $\mathrm{ROM}$ in the segments above an L4-L5 fusion. Shono et $\mathrm{al}^{19}$ investigated the effects of flexion/extension, lateral bending, and axial rotation on the level adjacent to spinal instrumentation. They found that when using transpendicular screws as fixation for 1 and 2-level instability, motion in the upper intact segment was significantly higher during axial rotation, flexion/extension, and lateral bending. In contrast to these reports, where rigid fixation was used, our simulation results predict that the mechanical interaction of the interspinous implant should not significantly alter the kinematics of the adjacent segments. After fusion, the increase in ROM at the adjacent level leads to an increased stress, possibly leading to degeneration. As a result, the device could be used as a transitional system between a fused and a totally free segment.

Our numerical results predict that the mechanical interaction of the implant with the spine segments unloads the disc better in extension than in flexion. If this estimate turns out realistic, the device could be indicated to treat patients suffering of low back pain caused by posterior overloading, in the early stage of the disc degeneration process. It could be used to treat pain due to posterior facet overloading, with associated initial disc height reduction, prolapse of posterior annulus often site of annular delamination, and/or nociceptive receptors after neo-vascularization. ${ }^{21}$ Then, it could be indicated to treat neurogenic claudication in the early stage of lumbar spinal stenosis. In fact, this syndrome is manifested by radicular pain, often bilateral, that is exacerbated by standing, walking, and other positions that place the lumbar spine in extension, ${ }^{22}$ while a flexed posture improves or relieves the symptoms. ${ }^{23,24}$

The presented model, as every computational model of complex phenomena, includes some simplifications to build a manageable model of the real structure. Living tissues biomechanics is extremely complex and not comprehensively investigated yet. As a result, a lot of modeling strategies were described in previous studies. The intervertebral disc, for example, has been included in the FE studies with several approaches: almost-incompressible solid elements ${ }^{25}$ or fluid elements for the nucleus pulposus ${ }^{26}$; anisotropic solid, fiber-reinforced composite or more complex continuum elements for the annulus fibrosus. ${ }^{27}$ In the present work, the nucleus pulposus was modeled as an almost-incompressible material, as suggested by Pitzen et al. ${ }^{9}$ The annulus fibrosus was modeled as a linear cylindrical orthotropic material. Even if the intervertebral disc is not exactly a cylindrical structure, the assumption of cylindrical orthotropy is not expected to strongly affect our simulation results.

Patients subjected to posterior dynamic stabilization present degenerated lumbar segments and thus, setting up a computational model including a degenerative state could be of clinical relevance. However, the spinal degeneration process is likely related to mechanical, biologic, and biochemical factors that can be hardly included in a purely mechanical model without strong assumptions. A possible approach is considering a specific degenerative state and neglecting the temporal evolution, by reducing disc height and modifying the mechanical properties of the nucleus pulposus. ${ }^{28}$ However, the mechanical properties of a degenerated disc are subjectspecific and so the needed mechanical parameters to model a degenerative state are difficult to define. For this reason, the present study focused on the biomechanics of the L3/S1 segment after implantation of an interspinous implant at a healthy level, targeting the work on the investigation of the biomechanics effect of the implant itself instead of its influence on a specific degenerative state.

A limitation of our FE model pertains to the assumed loading conditions. In fact, the manner in which the moments were applied in the current study corresponds to those usually considered in in vitro biomechanical studies of the lumbar spine. However, in vivo, the loads are transferred from the upper segments to the lower ones not only through the vertebral bodies but also through the posterior elements. Furthermore, the muscle loads, in the segment under consideration, have not been taken into account and the compressive loads have not been included. Therefore, the loadings considered in this study must be viewed as only an approximation of those occurring in vivo. Despite this simplifications, our results are consistent with those found in the literature.

Our study suggests that the DIAM spinal stabilization system may be able to share the load and to control the motion at the instrumented level, during flexion/extension. Anyway, no prospective, randomized, and multicentric clinical trial on this device has been reported yet, which is an essential procedure to evaluate any implant.

To understand the dynamic stabilization, a number of questions will need to be addressed by scientific investigations and careful study of clinical cases. In particular, it has not yet been analyzed how much load should be shared by the device and how much control of motion is desirable. These parameters need to be optimized by further studies to improve the clinical success of any dynamic stabilization device.

\section{REFERENCES}

1. Nockels RP. Dynamic stabilization in the surgical management of painful lumbar spinal disorders. Spine. 2005;15:S68-S72.

2. Mulholland RC, Sengupta DK. Rationale, principles and experimental evaluation of the concept of soft stabilization. Eur Spine J. 2002;11:S198-S205

3. Smith D, McMurry N, Disler P. Early intervention for acute back injury: can we finally develop an evidence-based approach? Clin Rehabil. 2002;16:1-11.

4. McNally DS, Shackleford IM, Goodship AE, et al. In vivo stress measurement can predict pain in discography. Spine. 1996; 21:2580-2587.

5. Crawford RP, Cann CE, Keaveny TM. Finite element models predict in vitro vertebral body compressive strength better than quantitative computed tomography. Bone. 2003;33:744-750.

6. Liebschner MAK, Kopperdhal DL, Rosenberg WS, et al. Finite element modeling of the human thoracolumbar spine. Spine. 2003;28:559-565. 
7. Ulrich D, Van Rietbergen B, Laib A, et al. The ability of threedimensional structural indices to reflect mechanical aspects of trabecular bone. Bone. 1999;25:55-60.

8. Lee KK, Teo EC, Fuss FK, et al. Finite-element analysis for lumbar interbody fusion under axial loading. IEEE Trans Biomed Eng. 2004;51:393-400.

9. Pitzen T, Geisles F, Matthis D, et al. A finite element model for predicting the biomechanical behaviour of the human lumbar spine. Control Eng Practice. 2002;10:83-90.

10. Eberlein R, Holzapfel GA, Fröhlich M. Multi-segment FEA of the human lumbar spine including the heterogeneity of the annulus fibrosus. Comput Mech. 2004;34:147-163.

11. Markolf KL. Deformation of the thoracolumbar intervertebral joints in response to external loads: a biomechanical study using autopsy material. J Bone Joint Surg Am. 1972;54:511-533.

12. Schultz AB, Warwick DN, Berkson MH, et al. Mechanical properties of human lumbar spine motion segments- Part I: responses in flexion, extension, lateral bending and torsion. J Biomech Eng. 1979;101:46-52.

13. Tencer AF, Ahmed AM, Burke DL. Some static mechanical properties of the lumbar intervertebral joint, intact and injured. J Biomech Eng. 1982;104:193-201.

14. Chen CS, Cheng CK, Liu CL, et al. Stress analysis of the disc adjacent to interbody fusion in lumbar spine. Med Eng Phys. 2001;23:483-491.

15. Lee CK. Accelerated degeneration of the segment adjacent to a lumbar fusion. Spine. 1988:13:375-377.

16. Schlegel JD, Smith JA, Schleusener RL. Lumbar motion segment pathology adjacent to thoracolumbar, lumbar, and lumbosacral fusions. Spine. 1996;21:970-981.

17. Lee CK, Langrana NA. Lumbosacral spinal fusion. A biomechanical study. Spine. 1984;9:574-581.
18. Chow DH, Luk KD, Evans JH, et al. Effects of short anterior lumbar interbody fusion on biomechanics of neighbouring unfused segments. Spine. 1996;21:549-555.

19. Shono Y, Kaneda K, Abumi K, et al. Stability of posterior spinal instrumentation and its effects on adjacent motion segments in the lumbosacral spine. Spine. 1998;23:1550-1558.

20. Yang SW, Langrana NA, Lee CK. Biomechanics of lumbosacral spinal fusion in combined compression-torsion loads. Spine. 1986; 11:937-941.

21. Lotz JC, Ulrich JA. Innervation, inflammation, and hypermobility may characterize pathologic disc degeneration: review of animal model data. J Bone Joint Surg Am. 2006;88:76-82.

22. Verbiest $\mathrm{H}$. A radicular syndrome from developmental narrowing of the lumbar vertebral canal. J Bone Joint Surg Br. 1954;36:230-237.

23. Dyck P, Doyle JB Jr. "Bicycle test" of van Gelderen in diagnosis of intermittent cauda equina compression syndrome. Case report. J Neurosurg. 1977;46:667-670.

24. Epstein JA, Epstein BS, Lavine LS, et al. Lumbar nerve root compression at the intervertebral foramina caused by arthritis of the posterior facets. $J$ Neurosurg. 1973;39:362-369.

25. Goel VK, Clausen JD. Prediction of load sharing among spinal components of a C5-C6 motion segment using the finite element approach. Spine. 1998;23:684-691.

26. Yoganandan N, Kumaresan SC, Liming Voo, et al. Finite element modeling of the C4-C6 cervical spine unit. Med Eng Phys. 1996;18:569-574.

27. Yin L, Elliott DM. A homogenization model of the annulus fibrosus. J Biomech. 2005;38:1674-1684.

28. Rohlmann A, Zander T, Schmidt H, et al. Analysis of the influence of disc degeneration on the mechanical behaviour of a lumbar motion segment using the finite element method. $J$ Biomech. 2005;39:2484-2490. 